\title{
WAVE RADIATION BY BALANCED MOTION IN A SIMPLE MODEL
}

\author{
J. VANNESTE*
}

\begin{abstract}
We introduce and study a toy model which captures some essential features of wave radiation by slow (or balanced) motion in the atmosphere and the ocean. Inspired by the widely studied five-component model due to Lorenz, the model describes the coupling of a nonlinear pendulum with linear waves. The waves obey a one-dimensional linear Klein-Gordon equation, so their dispersion relation is identical to that of inertia-gravity waves in a rotating shallow-water fluid. The model is Hamiltonian.

We examine two physically relevant asymptotic regimes in which there is some time-scale separation between the slow pendulum motion and the fast waves: in regime (i), the time-scale separation breaks down for waves with asymptotically large wavelengths; in regime (ii), the time-scale separation holds for all wavelengths. We study the generation of waves in each regime using distinct asymptotic methods. In regime (i), long waves are excited resonantly in a manner that is analogous to the Lighthill radiation of sound waves in weakly compressible flows, and to the radiation of gravitational waves by slow mass motion in general relativity. Matched asymptotics provides the functional form of the waves radiated, and leads, at higher order, to a closed model describing the pendulum dynamics while accounting for the dissipative effect of wave radiation. In regime (ii), an exponentially accurate slow manifold can be defined, and the waves radiated are exponentially small. They are captured using an exponential-asymptotic technique combining complex-time matching with Borel summation. The asymptotic results obtained in each regime are tested against numerical simulations of the model.
\end{abstract}

Key words. Slow manifold, wave radiation, inertia-gravity wave, exponential asymptotics

AMS subject classifications. 37N10, 76B15, 76U05, 37K05

1. Introduction. Systems with widely separated time scales abound, and numerous mathematical techniques have been devised to take advantage of their timescale separation. In many such systems, the fast degrees of freedom are only weakly excited; it is then natural to attempt to eliminate them [33] by reducing the dynamics to a slow manifold, that is, to a submanifold of the state space which is nearly invariant and on which the dynamics is slow. We refer the reader to the recent paper by MacKay [24] for a comprehensive discussion of the concept of slow manifold and for several examples of applications.

The particular application which motivates the present paper is provided by geophysical fluid dynamics. The dynamics of the atmosphere and the ocean at midlatitudes is dominated by the large-scale, slow motion usually referred to as 'balanced motion', but much faster motion in the form of inertia-gravity waves is also possible. (The even faster sound waves are generally filtered out at the outset by using incompressible, hydrostatic or anelastic fluid models.) The time-scale separation between the two types of motion is large, with typical time scales of the order of a few days or weeks for the balanced motion in the atmosphere or the ocean, respectively, and inertia-gravity-wave periods of the order of a few minutes. This has led to development of a variety of 'balanced models' describing the reduced dynamics on a slow manifold (see, e.g., [36, 5, 25] and references therein), the simplest of which is the well-known quasi-geostrophic model. Although balanced models are nowadays mostly theoreticians' tools, the concept of slow manifold is used in weather forecasting

\footnotetext{
*School of Mathematics, University of Edinburgh, King's Buildings, Edinburgh EH9 3JZ, UK (J.Vanneste@ed.ac.uk).
} 
in the process of initialization [9]: initial data are prepared by projection onto a slow manifold to reduce the level of (mostly spurious) inertia-gravity-wave activity.

The reliance on balanced models has led many researchers to investigate the fundamental limitations of the concepts of slow manifold and balance. This has been largely carried out using low-order models consisting of a few ordinary differential equations (ODEs), typically derived from the fluid equations by spectral expansion and severe truncation. The most widely studied among these is the five-component model due to Lorenz [21], also referred to as the Lorenz-Krishnamurthy (LK) model [22]. It can be reduced to four ODEs which describe the dynamics of a nonlinear pendulum, representing slow balanced motion, coupled to a stiff spring, representing the fast waves $[8,6]$. Another, essentially equivalent, model is the swinging spring [23], or spring pendulum [24].

For these ODE models, the status of the slow manifold is now well understood. In the absence of dissipation, thought to be negligible in the geophysical context, the slow manifolds are elliptic and in general not invariant [14, 24]. However, a systematic improvement procedure provides slow manifolds that are invariant up to an $O\left(\epsilon^{N}\right)$ error, where $\epsilon \ll 1$ is the ratio between the slow and fast time scales, for any $N \geq 1$. An optimal choice of $N$ then leads to an exponentially small error, under an assumption of analyticity $[14,24,38]$. The physical implications are clear: regardless of how well-prepared the initial data are, the generation of fast oscillations is unavoidable. For initial data lying on an optimal slow manifold, these oscillations are very weak, exponentially small in $\epsilon$. In the atmospheric context, this provides a mechanism for the generation of inertia-gravity waves, which is often referred to as 'spontaneous' generation, to emphasize the difference with the generation that results from the adjustment of poorly prepared initial data (see, e.g., [29] for a recent analysis).

In spite of the fact that their amplitude is beyond all orders in $\epsilon$, the fast oscillations generated spontaneously can be studied perturbatively, using the techniques of exponential asymptotics [32]. Such a study reveals the mechanism of generation to be an instance of the Stokes phenomenon (see, e.g., [3, 28]) and provides explicit estimates for the wave amplitudes. Results of this type have been obtained in [34] for the LK model and in [35, 27] for particular solutions of the fluid equations that are also governed by ODEs.

Low-order models such as the LK model have proved very useful for understanding the rather subtle questions raised by the concepts of slow manifold and balance. However, the drastic simplification entailed by the reduction from partial differential equations (PDEs) to ODEs means that a number of issues cannot be addressed using these models. To examine some of these issues, it is therefore useful to introduce a new simplified model, in the spirit of the LK and swinging-spring models, but retaining a PDE component. This is the purpose of this paper.

In fluids, the fast oscillations are propagating waves, with frequencies that depend on wavenumber. By fixing the wavenumbers involved, the derivation of LK-type models suppresses the possibility of interactions between very different wavenumbers. This possibility, however, is at the heart of one mechanism of wave generation which appears in several physical systems: gravitational waves in general relativity $[10,18$, $\S 110]$, sound waves in weakly compressible fluids [20, 19, §75], and, in the geophysical context, inertia-gravity waves in rotating shallow water $[11,12]$. In these systems, the wave frequencies decrease with wavenumbers in such a manner that there always are some resonant interactions between the slow motion and waves of sufficiently large 


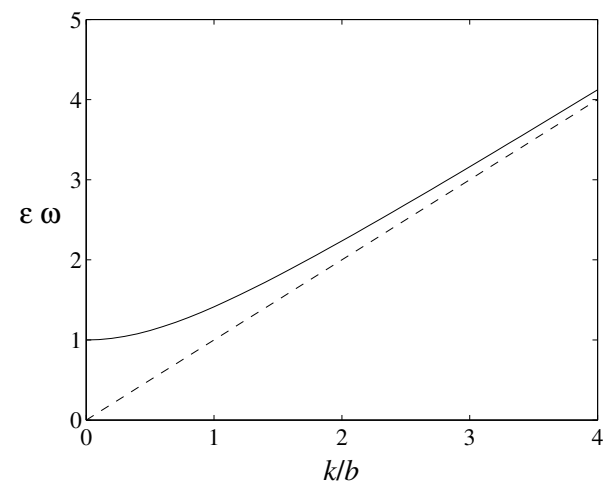

FIG. 1.1. Dispersion relation $\epsilon \omega=\left(1+k^{2} / b^{2}\right)^{1 / 2}$ for shallow-water inertia-gravity waves (solid line). The non-dispersive limit, valid for $k \gg b$, is also shown (dashed line).

scales. The wave generation is then relatively inefficient - because of the mismatch between the spatial scales of the slow motion and waves - but nevertheless scales like some power of the relevant small parameter rather than exponentially.

For the rotating shallow-water model and for more realistic models of geophysical flows, this resonant mechanism of wave generation (which we will refer to as Lighthill radiation following $[11,12]$ and the sound-wave analogy) or the non-resonant mechanism captured in the LK and other ODE models may be relevant, depending on the flow regime. To see why, consider the dispersion relation

$$
\omega^{2}=\epsilon^{-2}\left(1+k^{2} / b^{2}\right)
$$

of shallow-water inertia-gravity waves, displayed in Figure 1.1. Here, $\omega$ is the frequency and $k$ the wavenumber, and both are non-dimensionalized using the characteristic frequency $U / L$ and scale $L$ of the balanced motion. There are two independent parameters: the Rossby number, $\epsilon=U /(f L)$, where $f$ is the Coriolis parameter (measuring the earth's rotation rate), and the rotational Froude number $b=f L /(g h)^{1 / 2}$, where $g$ is the earth's gravity and $h$ the fluid depth. Two main asymptotic regimes are thought to be relevant: (i) the small-Froude-number regime, with $b \ll 1$ and $\epsilon=O(1)$, and (ii) the small-Rossby-number (or quasi-geostrophic) regime, with $\epsilon \ll 1$ and $b=O(1)$. In regime (i), there is no time-scale separation between (slow) balanced motion and long waves (with $O(b)$ wavenumbers), and Lighthill radiation occurs. In regime (ii), on the other hand, there is a time-scale separation between balanced motion and waves for all wavenumbers, since the wave frequency is bounded from below by $\epsilon^{-1} \gg 1$. Thus Lighthill radiation cannot occur, and one can expect exponentially small wave radiation of the type studied in the LK model [12, 31, 13].

The main advantage of the model that we introduce in this paper is that it makes it possible to analyze both regimes (i) and (ii) and, correspondingly, both types of wave generation in as simple a set-up as possible. The rotating shallow water may seem to be suitable for such an analysis: indeed, Ford, McIntyre and Norton [12] succeeded in capturing the Lighthill radiation and their feedback in regime (i) using matched asymptotics. This, however, requires a large amount of algebra which might deter many readers. Worse still, the asymptotic treatment of regime (ii) seems hopeless in the absence of a well-developed theory of exponential asymptotics for PDEs. By contrast, our model can be analyzed in regimes (i) and (ii) by relatively simple means. 
Another advantage of our model compared to low-order models is that, by keeping a PDE component, it introduces the possibility of wave radiation at infinity. Thus, the waves move away from their region of generation, thereby providing a source of dissipation for the balanced motion. This is probably a good approximation for what is happening in the atmosphere and ocean where the waves can escape before being ultimately damped by breaking or viscous dissipation. Our model can thus be used to examine how efficient wave generation and radiation can be as a mechanisms for the dissipation of the energy of the balanced motion. This is an issue of current interest in oceanography (see, e.g., [26]). It would be best studied by adding some forcing (perhaps random) to the model so that the properties of the statistical equilibrium arising from the balance between forcing and wave radiation can be established. In this paper, however, we limit our considerations to the unforced version of the model.

The new model is introduced in $\S 2$. It is a simple modification of the LK model in which the linear oscillator described by the fast variables is replaced by a linear, one-dimensional Klein-Gordon equation [37] with dispersion relation (1.1). Thus, the slow component of the model remains governed by ODEs, but the fast component is governed by PDEs. The coupling between the spatially dependent (fast) variables and the spatially independent (slow) variables is through an arbitrary localized shape function, which we take to be the derivative of a Gaussian. This has zero average and is odd so that, by symmetry, the spatial dynamics can be reduced the half line $\mathbb{R}_{+}$. The model is Hamiltonian. It is defined by three parameters: $\epsilon$ and $b$ which appear in the dispersion relation (1.1), and the amplitude $a$ of the shape function. The model is not derived in any way from the fluid equations, nor does it obviously represent any simple mechanical device. This is not a significant drawback, however. What is important for our purpose is that the parameters $\epsilon$ and $b$ play the same role as they do for the rotating shallow-water model. Because of this, we refer to them as the Rossby number and Froude number, respectively.

After introducing the model, we discuss the asymptotic behavior of its solutions. Section 3 is devoted to the small-Froude-number regime $b \ll 1, \epsilon=O(1)$. As mentioned above, this is the regime where Lighthill radiation occurs. Using matched asymptotics, we obtain an approximation for the waves generated spontaneously by the balanced motion. We further derive a reduced model, which describes the evolution of the slow variables whilst accounting for the energy loss due to wave radiation. We term this model 'post-balanced', by analogy with the post-Newtonian models used in general relativity to describe gravitational-wave radiation and its feedback on compact sources (see, e.g., [4] for a review). Our post-balanced model can then be seen as a toy version of the one derived by Ford et al. [12] for the rotating shallow-water equations.

Section 4 is devoted to the small-Rossby-number regime $\epsilon \ll 1$ and $b=O(1)$. This regime is similar to that studied in low-order models in that approximately invariant slow manifolds can be defined to arbitrary order $O\left(\epsilon^{N}\right)$, and wave radiation is exponentially weak. We estimate the amplitude of the waves radiated using exponential asymptotics. This shows, in particular, that the waves are near inertial, that is, have frequencies close to $\epsilon^{-1}$, with large spatial scales of the order of $\epsilon^{-1 / 2}$. The asymptotic results of both $\S 3$ and $\S 4$ are compared with numerical simulations. The numerical formulation, which implements non-reflecting boundary conditions, is described in Appendix A. The paper concludes with a Discussion in $\S 5$.

2. Model. The LK model [21, 22], obtained by truncation of a spectral expansion of the rotating shallow-water equations on the sphere, can be written as the 
system of five ODEs

$$
\begin{aligned}
\dot{u}_{\mathrm{L}} & =-v_{\mathrm{L}} w_{\mathrm{L}}+b v_{\mathrm{L}} y_{\mathrm{L}}, \\
\dot{v}_{\mathrm{L}} & =w_{\mathrm{L}} u_{\mathrm{L}}-b u_{\mathrm{L}} y_{\mathrm{L}} \\
\dot{w}_{\mathrm{L}} & =-u_{\mathrm{L}} v_{\mathrm{L}} \\
\delta \dot{x}_{\mathrm{L}} & =-y_{\mathrm{L}} \\
\delta \dot{y}_{\mathrm{L}} & =x_{\mathrm{L}}+b \delta u_{\mathrm{L}} v_{\mathrm{L}} .
\end{aligned}
$$

Here, the small parameter is $\delta$; it is related to the Rossby and Froude numbers by $\delta=\epsilon b /\left(1+b^{2}\right)^{1 / 2}$ so that both the small-Froude-number and small-Rossby-number regimes lead to $\delta \ll 1$. The slow variables $\left(u_{\mathrm{L}}, v_{\mathrm{L}}, w_{\mathrm{L}}\right)$ describe the evolution of a rigid body or, after reduction using the constancy of $u_{\mathrm{L}}^{2}+v_{\mathrm{L}}^{2}$, of a pendulum with $O(1)$ frequency. The fast variables $\left(x_{\mathrm{L}}, y_{\mathrm{L}}\right)$ describe a linear oscillator with frequency $\epsilon^{-1}[8,6]$.

We propose the following modification of the LK model. The three slow variables, which we denote by $(u, v, w)$, remain functions of $t$ only, but the fast variables, denoted by $(x, y)$ are functions of $t$ and of a spatial coordinate $s \in \mathbb{R}$. Choosing some localized function $f(s)$ (e.g. Gaussian or compactly supported), we write the new model as the mixed ODE-PDE system

$$
\begin{aligned}
\dot{u} & =-v w+v \int f(s) y(s, t) \mathrm{d} s, \\
\dot{v} & =w u-u \int f(s) y(s, t) \mathrm{d} s, \\
\dot{w} & =-u v \\
\epsilon x_{t} & =-y, \\
\epsilon y_{t} & =x-x_{s s} / b^{2}+\epsilon f(s) u v,
\end{aligned}
$$

where either $\epsilon$ or $b$ are now the small parameters. In (2.6)-(2.7) and in what follows, unspecified limits of integrations are $(-\infty, \infty)$. As announced, in the linear approximation, the fast variables $(x, y)$ satisfy a Klein-Gordon equation [37].

In what follows, we make the choice

$$
f(s)=\frac{a}{(2 \pi)^{1 / 2}} \frac{\mathrm{d}}{\mathrm{d} s} \mathrm{e}^{-s^{2} / 2}=\frac{-a s \mathrm{e}^{-s^{2} / 2}}{(2 \pi)^{1 / 2}},
$$

where $a$ is a fixed amplitude. Three qualitative properties of this function matter: the rapid decay as $|s| \rightarrow \infty$, the vanishing of its zeroth moment, and the non-vanishing of its first moment. Our results would be qualitatively the same for other choices of $f(s)$ satisfying these properties. The oddness of $f(s)$ is inessential, but convenient since it is inherited by $x(s, t)$ and $y(s, t)$ and allows computations to be limited to the half-line $s \in \mathbb{R}_{+}$.

Before examining solutions of (2.6)-(2.10), we reduce this system to four equations and mention some of its properties. Note first that the model conserves

$$
C=\frac{u^{2}+v^{2}}{2} \text { and } H=\frac{1}{2}\left(-u^{2}+w^{2}\right)+\frac{1}{2} \int\left(x_{s}^{2} / b^{2}+x^{2}+y^{2}\right) \mathrm{d} s .
$$

In fact, it is Hamiltonian and, when reduced to four equations, canonical (cf. [8, 6]). To see this, we first take $C=1$ without loss of generality. We then introduce the new 
coordinate $\phi$, with

$$
u=\sqrt{2} \cos \phi \quad \text { and } \quad v=\sqrt{2} \sin \phi
$$

and obtain the equations

$$
\begin{aligned}
\dot{\phi} & =w-\int f(s) y(s, t) \mathrm{d} s \\
\dot{w} & =-\sin (2 \phi) \\
\epsilon x_{t} & =-y \\
\epsilon y_{t} & =x-x_{s s} / b^{2}+\epsilon f(s) \sin (2 \phi)
\end{aligned}
$$

The further change of variables

$$
\theta=\phi-\epsilon \int f(s) x(s, t) \mathrm{d} s
$$

transforms the system into

$$
\begin{aligned}
\dot{\theta} & =w, \\
\dot{w} & =-\sin \left[2 \theta+2 \epsilon \int f(s) x(s, t) \mathrm{d} s\right], \\
\epsilon x_{t} & =-y, \\
\epsilon y_{t} & =x-x_{s s} / b^{2}+\epsilon f(s) \sin \left[2 \theta+2 \epsilon \int f(s) x(s, t) \mathrm{d} s\right] .
\end{aligned}
$$

This is a Hamiltonian system, with Hamiltonian

$$
H=\frac{w^{2}}{2}-\frac{1}{2} \cos \left[2 \theta+2 \epsilon \int f(s) x(s, t) \mathrm{d} s\right]+\frac{1}{2} \int\left(x_{s}^{2} / b^{2}+x^{2}+y^{2}\right) \mathrm{d} s
$$

and symplectic form

$$
\Omega=\mathrm{d} \theta \wedge \mathrm{d} \omega+\epsilon \mathrm{d} y \wedge \mathrm{d} x .
$$

Below we use the energy flux $F$ to diagnose the wave radiation. The flux emerges in the derivation of the conservation law for $H$ written in the form

$$
\frac{\mathrm{d} H}{\mathrm{~d} t}=-\int \partial_{s} F \mathrm{~d} s=0, \text { with } F=x x_{s} / b^{2} .
$$

In the form (2.15)-(2.18) the model can be recognized as describing the dynamics of a pendulum coupled nonlinearly with a Klein-Gordon wave equation, with dispersion relation (1.1). For waves with $O(1)$ wavenumbers, there is a time-scale separation between the slow pendulum and the wave motion if either $b \ll 1$ or $\epsilon \ll 1$. At leading order, a slow manifold is simply given $x=y=0$, and the corresponding balanced model corresponds to $(2.15)-(2.16)$ with $x=0$. The problem is then to derive reduced models, governing the slow evolution of $\theta$ and $w$ only, which are more accurate than this first-order balanced model. In the next two sections, we examine this problem for each of the two regimes $b \ll 1$ and $\epsilon \ll 1$. We use either of the two formulations (2.6)-(2.10) or (2.15)-(2.18), whichever is more convenient. 
3. Small- $b$ behavior. We start our asymptotic study of the new model by the small-Froude-number regime $b \ll 1$ and $\epsilon=O(1)$. As mentioned, the time-scale separation is not complete in this regime: long-wave solutions of the Klein-Gordon equation with $k=O(b)$ have an $O(1)$ frequency (see (1.1)) which can match the pendulum frequency. This is the origin of the Lighthill radiation which we now examine. Because this radiation appears at a small power of the small parameter $\left(O\left(b^{2}\right)\right.$ in the present case), it is easy to derive a post-balanced model, which reduces the dynamics to the two dependent variables $\theta$ and $w$ but nevertheless describes the effect of wave radiation. This asymptotic model, which we now derive, is analogous to the postNewtonian models developed for general relativity [4], and to the Ford et al. model for rotating shallow water [12].

3.1. Lighthill radiation and post-balanced model. Following [36], we expand only $x$ and $y$ in powers of the small parameter $b$, leaving equations (2.15)-(2.16) for $\theta$ and $w$ unexpanded. Introducing the expansion

$$
x=b^{2} x^{(0)}+b^{3} x^{(1)}+\cdots
$$

into (2.17)-(2.18) leads to

$$
x_{s s}^{(0)}=\epsilon f(s) \sin (2 \theta) \quad \text { and } \quad x_{s s}^{(1)}=0 .
$$

Solving and imposing oddness gives

$$
x^{(0)}=\epsilon F(s) \sin (2 \theta)+A(t) s \quad \text { and } \quad x^{(1)}=B(t) s,
$$

where

$$
F(s)=\frac{a}{(2 \pi)^{1 / 2}} \int_{0}^{s} \mathrm{e}^{-s^{\prime 2} / 2} \mathrm{~d} s^{\prime},
$$

and $A(t)$ and $B(t)$ are functions to be determined.

The expansion (3.1) breaks down for $s=O\left(b^{-1}\right)$. This reflects the breakdown of the time-scale separation for long waves with $k=O(b)$. In the outer region $s=$ $O\left(b^{-1}\right)$, we use the rescaled variable

$$
S=b s>0
$$

and expand

$$
x=b^{2} X^{(0)}(S, t)+b^{3} X^{(1)}(S, t)+\cdots
$$

Because of the rapid decay of $f(s)$ as $|s| \rightarrow \infty$, each $X^{(i)}, i=0,1, \cdots$ satisfies the free Klein-Gordon equation

$$
\epsilon^{2} X_{t t}^{(i)}-X_{S S}^{(i)}+X^{(i)}=0 .
$$

The solutions decaying as $S \rightarrow \infty$ are written in terms of their Laplace transforms in time. Denoting the Laplace transform by a tilde and the Laplace variable by $\sigma$, we find from (3.3) that

$$
\tilde{X}^{(i)}(S, \sigma)=\mathrm{e}^{-\left(1+\epsilon^{2} \sigma^{2}\right)^{1 / 2} S} \xi^{(i)}(\sigma),
$$


where the $\xi^{(i)}$ remain to be determined. Note that we have assumed vanishing initial conditions for $X$. Matching the Laplace transform of (3.2) with (3.4) and noting that $F(\infty)=a / 2$ gives

$$
A(t)=0, \quad \xi^{(0)}(\sigma)=\frac{a \epsilon}{2} \widetilde{\sin (2 \theta)}, \quad \tilde{B}(\sigma)=-\left(1+\epsilon^{2} \sigma^{2}\right)^{1 / 2} \xi^{(0)}(\sigma) \quad \text { and } \quad \xi^{(1)}(\sigma)=0 .
$$

Thus, to leading order, the waves generated by the balanced motion are given by

$$
\tilde{x}(s, \sigma) \approx b^{2} \tilde{X}^{(0)}(S, \sigma)=\frac{b^{2} a \epsilon}{2} \mathrm{e}^{-\left(1+\epsilon^{2} \sigma^{2}\right)^{1 / 2} S \widetilde{\sin (2 \theta)}} \text { for } S=b s=O(1) .
$$

This is also the result of a Lighthill-like approximation [20]: this takes advantage of the spatial scale separation between the waves and $f(s)$ to regard $(2.17)-(2.18)$ as a forced Klein-Gordon equation with the localized forcing $\epsilon f(s) \sin (2 \theta)$ approximated by $a \epsilon \delta^{\prime}(s) \sin (2 \theta)=b^{2} a \epsilon \delta^{\prime}(S) \sin (2 \theta)$. The feedback of the waves on $\theta$ and $w$ arises at $O\left(b^{3}\right)$, through the non-zero

$$
\tilde{B}(\sigma)=-\frac{a \epsilon\left(1+\epsilon^{2} \sigma^{2}\right)^{1 / 2}}{2} \widetilde{\sin (2 \theta)} .
$$

Inverting the Laplace transforms using the convolution theorem gives

$$
B(t)=-a \epsilon^{2} w \cos (2 \theta)-\frac{a \epsilon J_{1}(t / \epsilon)}{2 t} \star \sin (2 \theta),
$$

where $J_{1}(\cdot)$ is a Bessel function and $\star$ denotes convolution in time defined as

$$
\left(h_{1} \star h_{2}\right)(t)=\int_{0}^{t} h_{1}(t-\tau) h_{2}(\tau) \mathrm{d} \tau \text { for any two functions } h_{1}(t) \text { and } h_{2}(t) .
$$

Using (3.2), we compute

$$
\int f(s) x(s, t) \mathrm{d} s=-\frac{b^{2} a^{2} \epsilon}{2 \pi^{1 / 2}} \sin (2 \theta)-b^{3} a B(t)+O\left(b^{4}\right),
$$

and reduce $(2.15)-(2.16)$ to

$$
\begin{aligned}
\dot{\theta} & =w, \\
\dot{w} & =-\sin \left[2 \theta-b^{2} a^{2} \epsilon \sin (2 \theta) / \pi^{1 / 2}-2 b^{3} a \epsilon B(t)\right] .
\end{aligned}
$$

This is the sought post-balanced model. With $B(t)$ given in (3.5), it is a closed system of ordinary integro-differential equations for $\theta$ and $w$ which accounts for the effect of wave radiation. The model is not Hamiltonian because of the $O\left(b^{3}\right)$ term in (3.8). This term describes the loss of pendulum energy caused by the waves; its $O\left(b^{3}\right)$ scaling is consistent with the scaling of the wave-energy flux $F$. Estimated from the inner solution (3.2) as $s \rightarrow \infty$, the flux is found as

$$
F=x_{t} x_{s} /\left.b^{2} \approx b^{3} x_{t}^{(0)} x_{s}^{(1)}\right|_{s \rightarrow \infty}=b^{3} a^{2} \epsilon w \cos (2 \theta) B(t) .
$$

Note that since the system (3.7)-(3.8) is integrable when $b=0$, closed-form solutions for small $b$ could be derived by averaging. We do not pursue this here, since this possibility is a fragile particularity of our model. 

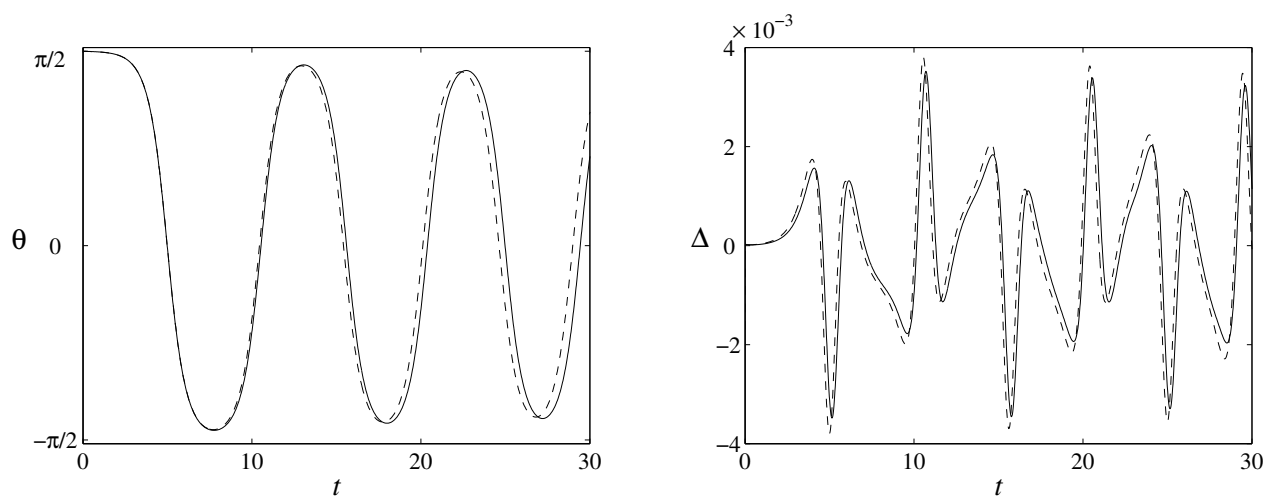

FIG. 3.1. Comparison of a numerical solution of the model (2.15)-(2.18) (solid curves) with the solution of the post-balanced approximation (3.7)-(3.8) derived in the limit $b \ll 1$ (dashed curves). The left panel shows the evolution of the angle $\theta$; the right panel compares the $O\left(b^{3}\right)$ quantity $\Delta$ defined in (3.11) with its post-balanced approximation $-b^{3} a B(t)$. The parameters chosen are $b=0.15, \epsilon=a=1$, and the initial conditions have been taken on the unperturbed heteroclinic trajectory (3.10).

A number of conclusions can be drawn from the above analysis. First, the wave radiation scales like a power, here the square, of the small parameter $b$. Next, the waves radiated are long, with $O\left(b^{-1}\right)$ wavelength, so that they can resonate with the slow pendulum motion. Because of this large scale, a Lighthill-like theory can be applied; this describe the waves as generated by a Dirac-type source which can be estimated by the first non-trivial moment (here the first) of the balanced part of the flow. Finally, the feedback of the waves on the flow can be captured asymptotically. This leads to a post-balanced model, which evolves on the slow time scale only and describes to leading order the impact of wave radiation on balanced motion. This model is dissipative, and involves time-integrals as well as derivatives, as a result of dispersion. These conclusions are identical (except for the specific scalings) to those that can be drawn from the analysis of the much more complex shallow water equations in [12].

3.2. Numerical results. We confirm the validity of the post-balanced approximation (3.7)-(3.8) by presenting the results of a numerical experiment. We compare the numerical solution of the full ODE-PDE model (2.15)-(2.18), implemented as described in Appendix A, with the numerical solution of (3.7)-(3.8) for particular initial conditions. These are chosen so that for $b=0$, the angle $\theta(t)$ follows the separatrix joining $\theta=\pi / 2$ to $\theta=-\pi / 2$. (We refer to this trajectory as heteroclinic, although it can also be viewed as homoclinic, if one identifies $\pi / 2$ with $-\pi / 2$ as is done in [8].) For our system, this unperturbed solution satisfies

$$
\cos \theta=\operatorname{sech}\left[\sqrt{2}\left(t-t_{0}\right)\right] \text { and } \sin \theta=-\tanh \left[\sqrt{2}\left(t-t_{0}\right)\right]
$$

for some $t_{0}$, which have taken to be $t_{0}=5$. When $b \neq 0$, the dissipation introduced by the wave radiation means that the heteroclinic trajectory is replaced by damped oscillations; with $b \ll 1$, these have long, slowly increasing periods.

This is illustrated by Figure 3.1 which shows the evolution of $\theta$ for $b=0.15$, with $\epsilon=a=1$. The period of the oscillations introduced by wave radiation is of the order of 10 . This is consistent with the order of magnitude $\log \left(b^{-3}\right)$ obtained by 

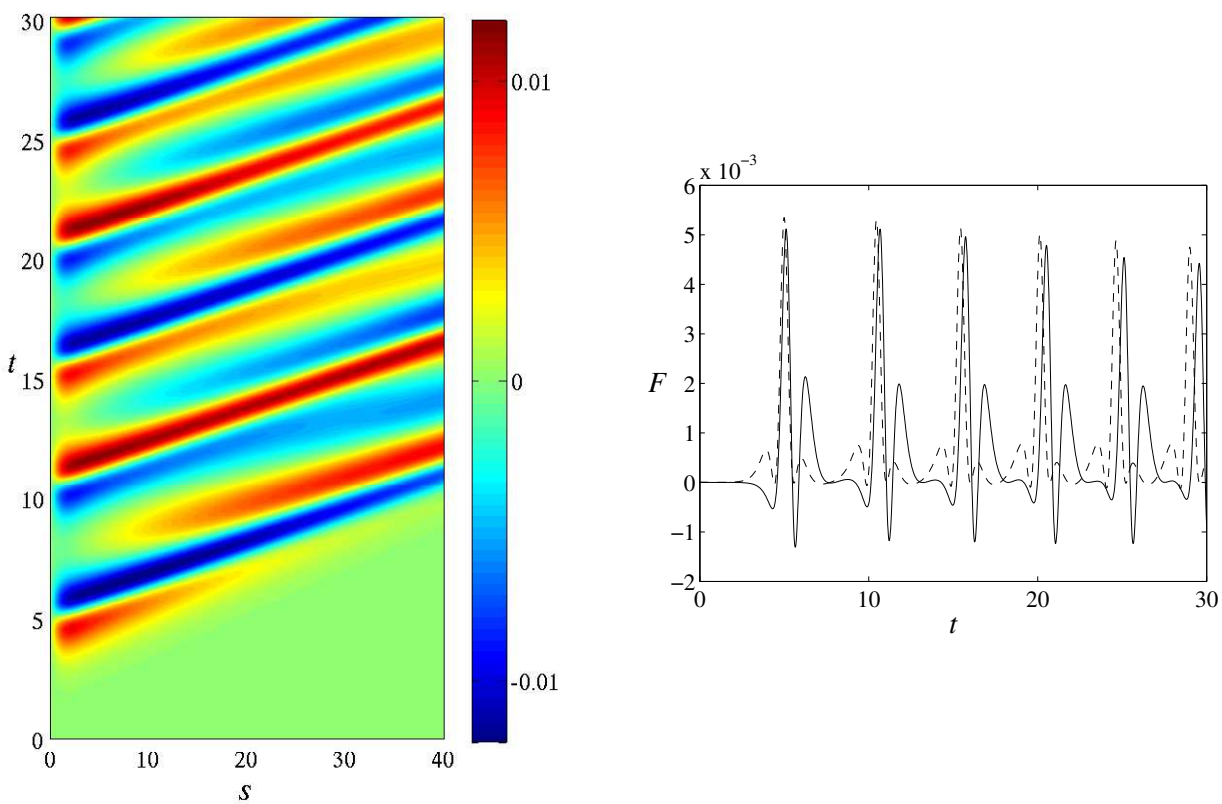

FIG. 3.2. Wave generation in the small b-limit. The left panel shows the evolution of $x(s, t)$, for the same parameters and initial conditions as in Figure 3.1. The right panel compares the energy flux $F=x_{s} x_{t} / b^{2}$ evaluated at $s=2$ (solid curve), with the post-balanced approximation (3.9) (dashed curved).

noting that the pendulum energy is decreased by an $O\left(b^{3}\right)$ amount from the separatrix energy. The figure compares the solution of the full model (solid curve) with that of the post-balanced model (dashed curve) and confirms the validity of the latter: the post-balanced approximation captures well the transition to damped oscillations, even though the period differs slightly from that obtained with the full model. The effectiveness of the post-balanced model is better judged from the right panel of Figure 3.1. This figure assesses the accuracy of the crucial approximation (3.6) by comparing

$$
\Delta=\int f(s) x(s, t) \mathrm{d} s+\frac{b^{2} a^{2} \epsilon}{2 \pi^{1 / 2}} \sin (2 \theta)
$$

with its approximation $-b^{3} a B(t)$. Because both functions are computed from the full-model solution, the phase shift present in the post-balanced solution disappears. The figure confirms that the validity of (3.6).

The left panel of Figure 3.2 shows the structure of the waves that are radiated from the balanced motion. As expected, these are long waves, with $O\left(b^{-1}\right)$ wavelengths. The right panel compares the flux (2.20) evaluated at $s=2$, with the flux (3.9) obtained in the asymptotic treatment. The chosen value $s=2$ is roughly consistent with the idea that (3.9) holds in an intermediate region between $s=O(1)$ and $s=$ $O\left(b^{-1}\right)$. If we factor out the phase shift mentioned above, there is a good agreement between the asymptotic and full numerical results. It is interesting to note that the actual flux changes sign while, by construction, the asymptotic one does not. There is a fair amount of cancellation in the actual flux which makes its time-integrated effect 
well described by the asymptotic one.

4. Small- $\epsilon$ behavior. We now turn to the regime $\epsilon \ll 1$, with $b=O(1)$, which in the shallow-water context, corresponds to the quasi-geostrophic regime. In this regime, there is a frequency separation between balanced motion and waves at all scales, and the wave generation can be expected to be exponentially weak. In this section, we use exponential asymptotics to estimate the amplitude of the waves generated.

4.1. Exponential asymptotics. A balanced solution of (2.15)-(2.18) can be sought by expansion of $\theta, w, x$ and $y$ in powers of $\epsilon$ or, alternatively, by first deriving an expression for a slow manifold in the form of slaving relations $x=x_{\text {bal }}(s, \theta, w)$ and $y=y_{\text {bal }}(s, \theta, w)$, and then reducing the dynamics onto it. The slaving relations can be derived order-by-order in $\epsilon$, through either an iteration or an expansion procedure. Choosing the latter, we write

$$
\begin{aligned}
& x_{\text {bal }}(s, \theta, w)=\sum_{n=0}^{\infty} \epsilon^{2 n+1} x_{\text {bal }}^{(n)}(s, \theta, w) \text { and } \\
& y_{\text {bal }}(s, \theta, w)=\sum_{n=0}^{\infty} \epsilon^{2 n+2} y_{\text {bal }}^{(n)}(s, \theta, w),
\end{aligned}
$$

and introduce these expressions into (2.15)-(2.18). This leads to a sequence of ODEs in $s$ for the $x_{\mathrm{bal}}^{(n)}(s, \theta, w)$ which are best solved in the Fourier domain. Denoting the Fourier transform in $s$ by a hat, with $k$ as the Fourier variable, we find

$$
\hat{x}_{\text {bal }}^{(0)}=-\frac{\hat{f}(k)}{\Omega^{2}} \sin (2 \theta), \quad \hat{y}_{\text {bal }}^{(0)}=\frac{2 \hat{f}(k)}{\Omega^{2}} w \cos (2 \theta), \text { etc. },
$$

where

$$
\hat{f}(k)=\frac{\mathrm{i} a k}{2 \pi} \mathrm{e}^{-k^{2} / 2}
$$

and $\Omega$ is the scaled frequency satisfying

$$
\Omega^{2}=\epsilon^{2} \omega^{2}=1+k^{2} / b^{2} .
$$

The Fourier transform can be inverted. For instance, we find that

$$
x_{\text {bal }}^{(0)}=-\frac{a b^{2} \mathrm{e}^{b^{2} / 2}}{4}\left(\mathrm{e}^{b s} \operatorname{erfc} \frac{b+s}{\sqrt{2}}-\mathrm{e}^{-b s} \operatorname{erfc} \frac{b-s}{\sqrt{2}}\right) \sin (2 \theta) .
$$

By contrast with the small- $b$ case, there are no obstacles to carrying out this calculation to obtain, in principle, $x_{\text {bal }}^{(N)}$ and $y_{\text {bal }}^{(N)}$ for arbitrary order $N$. The decay of $x_{\mathrm{bal}}^{(N)}$ and $y_{\mathrm{bal}}^{(N)}$ as $|s| \rightarrow \infty$, in particular, is satisfied. Indeed a rough estimate indicates that $\hat{x}^{(N)} \propto \hat{f}(k) / \Omega^{N+1}$, leading to

$$
x^{(N)} \propto s^{N} \mathrm{e}^{-b|s|} \text { as }|s| \rightarrow \infty .
$$

Of course, the series (4.1) diverge, and only finite values of $N$, typically up to $O\left(\epsilon^{-1}\right)$, can be considered. This divergence reflects the existence of a subdominant solution 

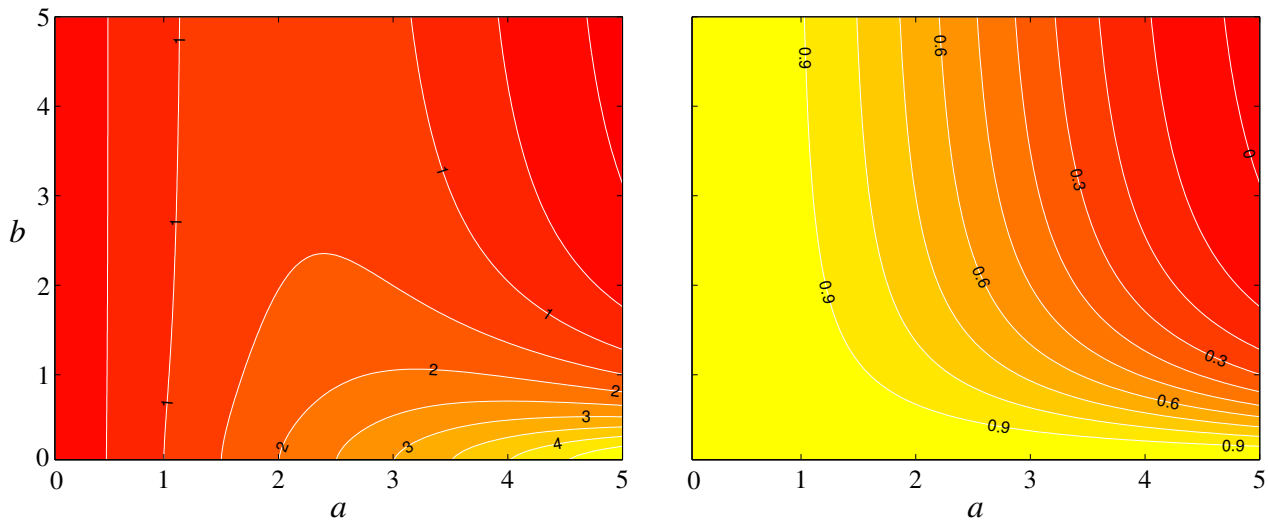

FIG. 4.1. Value of $\lambda(0)$, governing the amplitude of the waves generated spontaneously in the limit of small $\epsilon$, as a function of $a$ and $b$ (left panel). The right panel shows $\lambda(0) / a$ and demonstrates that $\lambda(0) \sim a$ for $a \rightarrow 0$ or $b \rightarrow 0$.

which is switched on through a Stokes phenomenon $[1,3]$. Physically, this subdominant solution represents waves, and the switching-on corresponds to their spontaneous generation by the balanced motion. Indeed, introducing the decomposition $x=x_{\text {bal }}+x_{\mathrm{w}}$, with $x_{\text {bal }}$ defined by an optimally truncated series of the form (4.1) and $x_{\mathrm{w}} \ll x_{\mathrm{bal}}$, we find that $x_{\mathrm{w}}$ satisfies the free Klein-Gordon equations at leading order in $\epsilon$. Thus, the time dependence of the Fourier transform of $\hat{x}_{\mathrm{w}}$ is

$$
\hat{x}_{\mathrm{w}}(k, t) \propto \exp (\mathrm{i} \omega t),
$$

where the branch of $\omega$ defined by (1.1) is chosen for radiation at $|s| \rightarrow \infty$. If the solution is properly balanced, then $\hat{x}_{\mathrm{w}}=0$. However, in the course of the evolution, a Stokes phenomenon can occur which switches $\hat{x}_{\mathrm{w}}$ to an exponentially small, non-zero value which we estimate.

The Stokes phenomenon is associated with singularities of the balanced motion for complex values of $t$. By analyzing the equations near the relevant singularities, and matching with expressions (4.1) and (4.7) which are valid some distance away from these singularities, one can estimate $x_{\mathrm{w}}$. This is the essence of the so-called KruskalSegur method, which we apply in Appendix B. There, we obtain the amplitude of the waves switched on when the (real) time $t$ crosses the Stokes line joining a pair of complex-conjugate singularities $t_{*}$ and $\bar{t}_{*}$. In terms of Fourier transform, we find

$$
\hat{x}_{\mathrm{w}}(k, t) \sim \frac{-\mathrm{i} k \lambda(k)}{\epsilon} \mathrm{e}^{-\alpha \omega / \epsilon-k^{2} / 2} \cos [\omega(t-\beta) / \epsilon],
$$

where

$$
\alpha=\operatorname{Im} t_{*}>0 \text { and } \beta=\operatorname{Re} t_{*} .
$$

In this expression, $\lambda(k)$ is an $O(1)$ function defined for small $k$ that can be obtained numerically as described in Appendix B by solving the discretized version of an infinite set of recurrence relations. The switching on takes place for $t=\beta$ so that (4.8) needs to be multiplied by the Heaviside function $H(t-\beta)$ (which could be smoothed out as an error function of width $\epsilon^{1 / 2}$ using Berry's result [3]), and only the singularities $\left(t_{*}, \bar{t}_{*}\right)$ nearest to the real axis need to be taken into account. 
The Fourier transform can be inverted to derive an approximation for $x_{\mathrm{w}}(s, t)$ from (4.8); using the steepest-descent method, we find the final estimate

$$
x_{\mathrm{w}}(s, t) \sim(2 \pi)^{1 / 2} b^{3} \lambda(0) \mathrm{e}^{-\alpha / \epsilon} S \operatorname{Re} \frac{\mathrm{e}^{\mathrm{i}(t-\beta) / \epsilon-b^{2} S^{2} /[2(\alpha-\mathrm{i}(t-\beta))]}}{[\alpha-\mathrm{i}(t-\beta)]^{3 / 2}},
$$

valid for $S=\epsilon^{1 / 2} s=O(1)$. This provides a useful closed-form approximation which requires only to compute the value of $\lambda(0)$. Figure 4.1 shows $\lambda(0)$ as a function of $a$ and $b$; it also shows $\lambda(0) / a$ to demonstrate that $\lambda(0) \sim a$ for $a \rightarrow 0$ or $b \rightarrow 0$. In fact, it can be established that these limits correspond to the decoupling of $\theta$ and $w$ from $x$ and $y$, and that

$$
\lambda(k) \sim a \text { as } a \rightarrow 0 \text { or } b \rightarrow 0 .
$$

Formula (4.9) shows that the waves emitted are 'near-inertial' waves, that is, have a frequency close to the minimum frequency $\epsilon^{-1}$, and have large, $O\left(\epsilon^{-1 / 2}\right)$ spatial scales.

It is interesting to examine what a Lighthill-like approach would predict for the wave amplitude in the regime $\epsilon \ll 1$. This approach amounts to solving (2.17)(2.18), with the last term of (2.18) approximated by $\epsilon \sin \left(\theta^{(0)}\right)$, where $\theta^{(0)}$ is the leading-order approximation to $\theta$. The computation of the wave radiated in this case is straightforward in Fourier space, where it follows the computation carried out originally for the LK model in [22]. The result has the form (4.8), with $\lambda(k)$ replaced by $a$, consistent with (4.10). For finite $a$ and $b, \lambda(k)$ differs significantly from $a$ (see Figure 4.1). Thus, a Lighthill-like approach does not give the correct asymptotics for amplitude of the Fourier modes even to leading order (although the controlling behavior, that is, the asymptotics of the log of the amplitude is correct). This is because the wave amplitude at leading order in $\epsilon$ depends on the structure of the balanced motion to all orders. Thus, in contrast with the small- $b$ situation, an essentially complete description of the balanced motion is necessary to estimate even the leading-order wave amplitude. We say 'essentially' here, because this complete description is in fact needed only in the vicinity of the singular points $t_{*}$ nearest to the real axis rather than for all $t$. This is the simplification that is exploited in the asymptotic approach of Appendix B.

4.2. Numerical results. We compare the theoretical predictions of exponential asymptotics with numerical results for two sets of parameters. For the first, we choose the same initial condition as in $\S 3.2$, that is, on the unperturbed $(\epsilon=0)$ heteroclinic trajectory (3.10). For this trajectory, there is only one pair of complex-conjugate singularities of the balanced motion, $t_{*}$ and $\bar{t}_{*}$ with

$$
t_{*}=t_{0}+\mathrm{i} \frac{\pi}{2 \sqrt{2}}
$$

so that $\alpha=\pi /(2 \sqrt{2})$ and $\beta=t_{0}$. An initial condition on the heteroclinic trajectory makes the wave generation particularly easy to identify and avoids the difficulties associated with the initialization of the fast variables $(x, y)$. By taking $t_{0}$ sufficiently large, all the time derivatives of $w$ and $\theta$ at $t=0$ can be made arbitrarily small, leading to arbitrarily small values for the coefficients $x_{\mathrm{bal}}^{(i)}, i>0$, and $y_{\mathrm{bal}}^{(i)}, i \geq 0$, of the balanced part of $x$ and $y$. Thus, taking

$$
x(0)=\epsilon x_{\text {bal }}^{(0)}, \quad y(0)=0,
$$




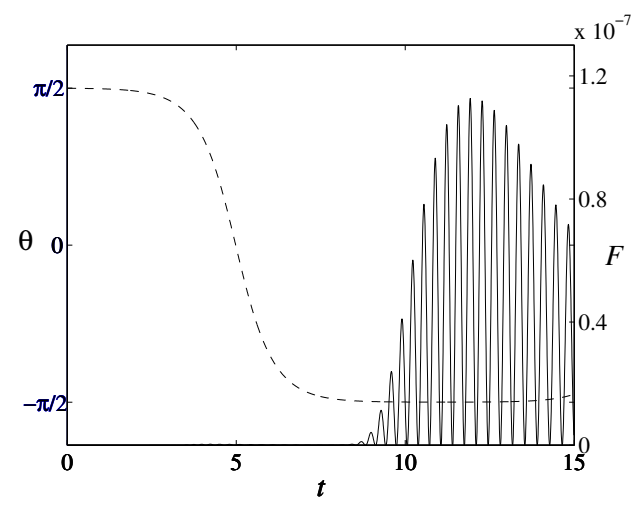

FIG. 4.2. Wave generation for $\epsilon=0.125$, with $a=2$ and $b=1$ for initial conditions on the unperturbed heteroclinic trajectory. The angle $\theta$ (dashed curve, left axis) and wave energy flux $F$ at $s=25$ (solid curve, right axis) are shown as a function of time.

where $x_{\mathrm{bal}}^{(0)}$ is given by (4.5) with $\theta=\theta(0)$, provides a solution that can be balanced to an arbitrary accuracy simply by taking $t_{0}$ large enough. We have taken $t_{0}=5$ which turns out to be sufficient to make the wave radiation by initial adjustment negligible.

Figure 4.2 shows the evolution of $\theta$ obtained for the parameter choice $\epsilon=0.125$, $a=2$ and $b=1$. As expected, $\theta$ follows closely the heteroclinic trajectory; it does not tend to $\pi / 2$ for large $t$, however, and a longer simulation would reveal that $\theta$ undergoes a series of weakly damped oscillations. The damping is of course associated with the wave radiation. This is first diagnosed by showing the wave-energy flux $F$ computed at the boundary $s=25$ of our integration domain. The flux is always positive, confirming the proper implementation of the radiation boundary condition, and it oscillates rapidly, with a frequency $2 / \epsilon$; its maximum value, of the order of $10^{-7}$, is consistent with the crude estimate $\exp (-2 \alpha / \epsilon) \approx 2 \times 10^{-8}$ which follows from (4.9).

A more complete picture of the wave radiation emerges from Figure 4.3. This shows $x-x_{\mathrm{bal}}^{(0)}$ as a function of $s$ and $t$. The variable $x-x_{\mathrm{bal}}^{(0)}$ includes an $O\left(\epsilon^{3}\right)$ contribution of the balanced part of $x$ as well as the exponentially small wave part $x_{\mathrm{w}}$. However, the balanced contribution decreases rapidly for $t>t_{0}$ and for $s>1$, so that the wave contribution is clearly isolated. This consists of rapidly propagating waves, with frequency approximately equal to $\epsilon^{-1}$, that are emitted for $t \approx t_{0}=5$ from the region $s=O(1)$. The right panel of Figure 4.3, showing the estimate (4.9) for $x_{\mathrm{w}}$ confirms the validity of our asymptotic treatment. Note that for the chosen parameters $a=2$ and $b=1$, we obtain $\lambda(0)=1.69$ using the method described in Appendix B; a Lighthill-like approach would therefore overestimate the amplitude of the wave generation by the factor $a / \lambda(0) \approx 1.2$.

For the second numerical experiment, we have chosen an initial condition on a periodic trajectory of the undisturbed system. The periodic trajectories can be written explicitly as

$$
\theta=-\operatorname{am}\left(\sqrt{2}\left(t-t_{0}\right) / k, k\right)
$$

where am is the amplitude of the Jacobian elliptic functions [2, Ch. 16], and $k \geq 1$ and $t_{0}$ are fixed by the initial conditions. The period of (4.11) is $2 \sqrt{2} k K(k)=2 \sqrt{2} K(1 / k)$, where $K$ is the elliptic integral of the first kind. The solutions (4.11) have complex- 



FIG. 4.3. Wave generation for the same parameters as in Figure 4.2. The $O\left(\epsilon^{3}\right)$ quantity $x-x_{\mathrm{bal}}^{(0)}$ (left panel) is compared with the asymptotic result (4.9) for the wave part $x_{\mathrm{w}}$ (right panel). The color code, used for both panels, does not cover the large values of $\left|x-x_{\text {bal }}^{(0)}\right|$ for $4<t<6$ which reach $1.8 \times 10^{-3}$

time poles, with the nearest to the real axis located at

$$
t_{* n}=t_{0}+n \sqrt{2} K(1 / k)+\mathrm{i} K^{\prime}(1 / k) / \sqrt{2} \text { and } \bar{t}_{* n}, \text { where } n \in \mathbb{Z}
$$

and $K^{\prime}$ is the complementary elliptic integral. We have taken the parameters $\epsilon=0.15$, $a=2.5$ and $b=0.5$. For initial conditions we have chosen $\theta=\pi / 4$ and $w=0$, giving

$$
k=\sqrt{2}, \quad t_{0}=K(1 / \sqrt{2}) / \sqrt{2}=1.311 \cdots,
$$

and poles $t_{* n}=(2 n+1+\mathrm{i}) t_{0}$. Correspondingly, for real $t$, Stokes lines are crossed for $t=t_{n}=(2 n+1) t_{0}$, when the unperturbed trajectory has vanishing angle $\theta$.

For periodic unperturbed solutions, it is difficult to compute balanced initial conditions accurately: in principle, there is no alternative to the computation of $x_{\text {bal }}$ and $y_{\text {bal }}$ by optimally-truncated series expansion. In practice, however, we found that the lack of balance that results from truncating these series to $O(\epsilon)$, that is, from taking $x=\epsilon x_{\mathrm{bal}}^{(0)}$ given in (4.5) with $\theta=\pi / 4$, and $y=0$ is not problematic. A small-amplitude wavepacket is emitted at the initial time which leaves quickly the computational domain while adjusting the solution to a well-balanced state.

Figure 4.4 shows the evolution of $\theta$ and of the wave-energy flux $F$ at the boundary of our computational domain, taken to be $s=50$. The angle $\theta$ oscillates according to (4.11), with small corrections. The flux $F$ has a large peak for $t \approx 4$ that corresponds to the passage of the wavepacket emitted by the initial adjustment. Thereafter, the flux is associated with the exponentially small waves radiated spontaneously when $t$ 


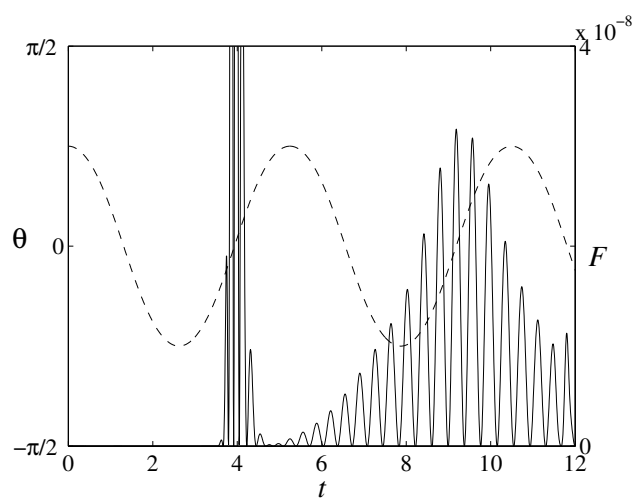

FIG. 4.4. Wave generation for $\epsilon=0.15$, with $a=2.5$ and $b=0.5$ for initial conditions on a periodic unperturbed trajectory. Angle $\theta$ (dashed curve, left axis) and wave energy flux $F$ at $s=50$ (solid curve, right axis) as a function of time.
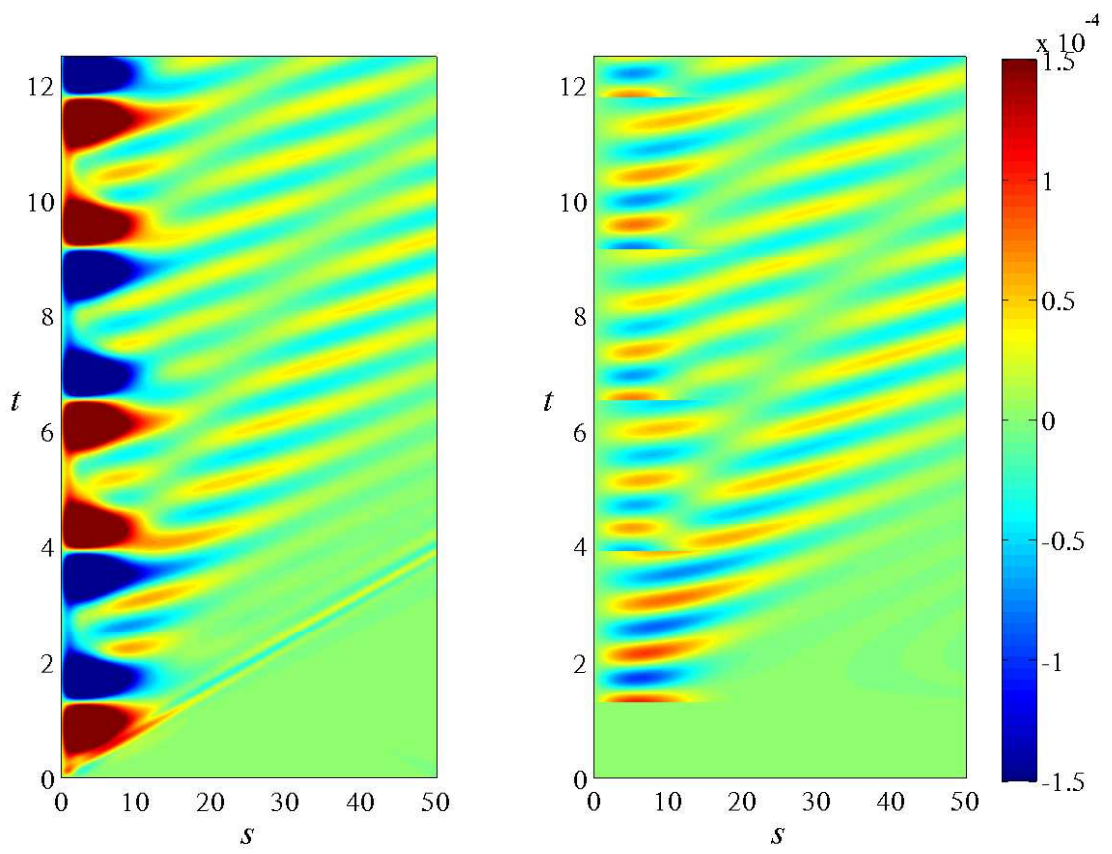

FIG. 4.5. Wave generation for the same parameters as in Figure 4.4. The $O\left(\epsilon^{3}\right)$ quantity $x-x_{\mathrm{bal}}^{(0)}$ (left panel) is compared with the asymptotic result (4.9) for the wave part $x_{\mathrm{w}}$ (right panel). The color code, used for both panels, does not cover the large values of $\left|x-x_{\mathrm{bal}}^{(0)}\right|$ for $0 \leq s<10$ which reach $5.8 \times 10^{-4}$.

crosses Stokes lines. The estimate $\exp (-2 \alpha / \epsilon)$, with $\alpha=\operatorname{Im} t_{* n}=t_{0}=1.311 \cdots$, gives $2.5 \times 10^{-8}$, in rough agreement with the observed flux.

The left panel of Figure 4.5 shows $x-x_{\mathrm{bal}}^{(0)}$ as a function of $s$ and $t$. Although this field is dominated by the balanced component for $s=O(1)$, the exponential decay of this component with $s$ (see (4.6)) ensures that the wave part emerges for large $s$, say 
for $s>10$. After the wavepacket created by the initial adjustment leaves the domain, the wave field consists of a superposition of large-scale wavepackets emitted at $t=t_{n}$, creating an interference pattern. The right panel of Figure 4.5 shows the asymptotic prediction for $x_{\mathrm{w}}$. To construct the figure, we have used (4.9), with $\alpha=\operatorname{Im} t_{* 0}=1.311$ and $\beta=\operatorname{Re} t_{* 0}=1.311$ and $\lambda(0)=2.235$ to estimate the wave radiated when the first Stokes line is crossed, at $t=t_{0}$. The full wave field is then the superposition of similar contributions generated for each $t_{n}$. If we denote the first contribution by $x_{\mathrm{w} 0}\left(s, t-t_{0}\right)$, the full wave field is given by

$$
x_{\mathrm{w}}(s, t)=\sum_{n=0}^{\infty}(-1)^{n} x_{\mathrm{w} 0}\left(s, t-t_{n}\right) H\left(t-t_{n}\right),
$$

where $H(\cdot)$ denotes the Heaviside function, and the factors $(-1)^{n}$ appear because the signs of the balanced variables near the poles $t_{* n}$ alternate with $n$. On the whole, this asymptotic expression gives a good picture of the wave field, even though the amplitudes are somewhat overestimated, and the interference pattern appears slightly distorted. However, keeping in mind that (4.9) has an $O(\epsilon)$ error and is only valid for $s=O\left(\epsilon^{1 / 2}\right)$, the agreement between the asymptotic prediction and the numerical result is satisfactory.

5. Discussion. In this paper, we introduce the simple mixed ODE/PDE system (2.15)-(2.18) as a toy model for wave radiation by slow (or balanced) motion in the atmosphere and ocean. Though no more than a caricature of the real atmosphere and oceans, the model has a number of appealing features: wave dispersion relation identical to that of shallow-water inertia-gravity waves, Hamiltonian structure, possibility of wave radiation to infinity, and, of course, great simplicity. Compared to low-order models such as the LK and swinging-spring models, the inclusion of a PDE component is a major advance toward realism. It makes it possible to examine the interaction between waves and balanced motion of vastly different spatial scales that is at the core of the Lighthill-like radiation (or gravitational-wave-like) radiation in the small-Froude-number regime. The simplicity of the model also allows the estimation of wave radiation in the small-Rossby-number regime using exponential asymptotics. It emerges from the analysis that the wave amplitude in the latter regime cannot be estimated as they are in the former regime, by simply regarding the wave equation as forced by terms computed from the leading-order balanced motion. Indeed, estimating the wave amplitude in the small-Rossby-number regimes requires knowledge of the balanced motion to all algebraic orders in $\epsilon$, at least near complex-time singularities. This makes the study of spontaneous wave generation in the more realistic context of, say, the shallow-water model particularly challenging.

Another phenomenon that the PDE component makes it possible to represent is the loss of energy through wave radiation. This is of great interest since some form of dissipation is key to the maintenance of balance in the atmosphere and ocean. This dissipation is poorly modeled by simply adding a small damping to the fast equations, however, because the fast variables are in fact dominated by their balanced, slow component which thus becomes significantly affected by dissipation. In real fluids, however, dissipation is thought to affect mostly the truly fast component of the dynamics, through wave radiation and possibly nonlinear forward energy cascades. The new model realistically reproduces at least the first of these processes, and it could be used to investigate how close to slow manifolds the evolution of the system remains owing to wave radiation. Discussing these aspects will of course involve typical PDE 
issues, such as the choice of a norm suitable to measure wave activity in the vicinity of the origin. It is worth noting that the energy dissipation caused by wave radiation does not necessarily imply that the balanced system relaxes to a state of rest. In fact, it is not even the case for our model, when formulated in terms of the original slow variables $(u, v, w)$, since the ultimate state $w=0$ and $\theta=0$ corresponds to $u=\sqrt{2}$, $v=0, w=0$. One can interpret this by recognizing that the conservation of $u^{2}+v^{2}$, left unaffected by wave radiation, prevents the system from relaxing to a resting state. This observation is trivial for our model, but less so for the fluid systems: for these the relaxation will be toward a state of minimum energy for a fixed distribution of potential vorticity. Since this state is typically not smooth, this means that wave radiation leads to the formation of complicated, rough structures.

There are several generalizations of the model introduced in this paper which it may be worthwhile to consider. A first, mentioned in the Introduction, is the addition of a slow forcing. The aim of this addition is to study the statistical equilibrium that can emerge from the balance between forcing and radiation. A second modification is to alter the system so as to make the balanced dynamics chaotic. This is useful to remove some of the peculiarities that arise because of the integrability of the onedegree-of-freedom balanced system. Wirosoetisno and Shepherd [39] introduced a modification of the LK model which amounts to replacing the constant value of $u_{\mathrm{L}}^{2}+v_{\mathrm{L}}^{2}$ by an arbitrarily chosen function of time. Our model could be modified in exactly the same way.

We conclude by noting that the study of wave radiation by balanced motion need not be limited to the regimes of small Froude number $b$ or small Rossby number $\epsilon$ as is the case here. Although balance is difficult to define in the absence of a small parameter, it is often remarked that the qualitative features observed for small $b$ or $\epsilon$, in particular the clear separation between wave and flow-type motion, persist when these parameters are of order unity. The model introduced in this paper could very well be used as a starting point to examine this issue and to give a precise meaning to, as well as an explanation for, this observation.

Acknowledgments. The author is funded by a NERC Advanced Research Fellowship. He thanks D. Wirosoetisno for useful comments on the manuscript.

\section{Appendix A. Numerical implementation.}

We need to solve numerically the model equations $(2.15)-(2.16)$ in a finite domain $[-L, L]$ in manner that allows outward radiation of wave energy. To this end, we implement exact non-reflecting boundary conditions. This takes advantage of the fact that $f(s)$ is localized, so that, for $|s| \gg 1,(2.17)-(2.18)$ reduce to the linear Klein-Gordon equation and can be solved in closed form.

Consider the solution of the Klein-Gordon equation in $[L, \infty)$ with radiation boundary condition as $s \rightarrow \infty$. In term of the Laplace transform $\tilde{x}(s, \sigma)$ of $x(s, t)$, where $\sigma$ is the Laplace variable, this solution reads

$$
\tilde{x}(s, \sigma)=\exp \left[-b\left(1+\epsilon^{2} \sigma^{2}\right)^{1 / 2} s\right] \xi(\sigma),
$$

for some function $\xi(\sigma)$. This gives the simple Dirichlet-to-Neuman map [15]

$$
\tilde{x}_{s}(s, \sigma)=-b\left(1+\epsilon^{2} \sigma^{2}\right)^{1 / 2} \tilde{x}(s, \sigma) .
$$

Upon transforming back to the time domain and evaluating at $s=L$, this reads

$$
x_{s}(L, t)=-b \epsilon x_{t}(L, t)-b \frac{J_{1}(t / \epsilon)}{t} \star x(L, t),
$$


where $J_{1}(\cdot)$ is a Bessel function, and $\star$ denotes convolution in time. This provides an exact non-reflecting condition at $s=L$ for the Klein-Gordon equation; we employ it for our system with $L$ large enough that $f(L)$ is negligible.

We solve the model equations (2.15)-(2.18) by finite differences, using a uniform grid for $s \in[0, L]$ and a Störmer-Verlet scheme both in $t$ and $s$. This discretization is symplectic for the ODE part of the model, and multi-symplectic for the PDE part [7]. The boundary condition (A.1) is discretized in a straightforward manner, using a backward difference for the $s$ - and $t$ - derivatives, and the trapezium rule for the convolution. This discretization of the exact boundary condition can lead to spurious wave reflection at $s=L[30]$, but we have found this to be negligible.

\section{Appendix B. Exponential asymptotics for $\epsilon \ll 1$.}

In this Appendix, we use exponential asymptotics to obtain the estimates (4.8)(4.9) for the amplitude of the waves generated by the balanced motion in the regime $\epsilon \ll 1, b=O(1)$. The method is similar to that used in [34] for the LK model, and we refer the reader to that paper for further details.

The balanced solutions of (2.15)-(2.16) may be obtained by expansion in powers of $\epsilon$ of all the variables. Because it is convenient to use the formulation (2.6)-(2.10), we expand

$$
u(t)=\sum_{n=0}^{\infty} \epsilon^{n} u^{(n)}(t) \text { and similarly for } v \text { and } w
$$

and

$$
x(s, t)=\sum_{n=0}^{\infty} \epsilon^{n+1} x^{(n)}(s, t) \text { and } y(s, t)=\sum_{n=0}^{\infty} \epsilon^{n+2} y^{(n)}(s, t) .
$$

These series, whose coefficients can be computed recursively, describe the balanced part of the solution. The leading-order coefficients $u^{(0)}(t), v^{(0)}(t)$ and $w^{(0)}(t)$, in particular, solve

$$
\dot{u}^{(0)}=-v^{(0)} w^{(0)}, \quad \dot{v}^{(0)}=w^{(0)} u^{(0)}, \quad \dot{w}^{(0)}=-u^{(0)} v^{(0)},
$$

and are given in terms of Jacobi elliptic functions. The wave part of the solution is exponentially smaller, and to leading order given by the solution $x_{\mathrm{w}}(s, t)$ of a KleinGordon equation. In terms of Fourier transform, this reads

$$
\hat{x}_{\mathrm{w}}(k, t)=C(k) \mathrm{e}^{\mathrm{i} \omega t}+\text { c.c. },
$$

where $C(k)$ is a Stokes multiplier switching from zero to an exponentially small value as a Stokes line is crossed by $t$.

The coefficients in (B.1)-(B.2) have singularities in the complex plane near which the expansions (B.1)-(B.2) cease to be valid. In the vicinity of these singularities, the balanced and wave parts of the solution are not well separated, and (B.1)-(B.2), which we can regard as an outer solution, must be replaced by a different, inner, solution. We now consider this inner solution near a singularity $t_{*}$ with $\operatorname{Im} t_{*}>0$.

The behavior of the outer solution near $t_{*}$ is readily obtained as

$$
u \sim \frac{-\mathrm{i}}{t-t_{*}}, v \sim \frac{-1}{t-t_{*}}, w \sim \frac{\mathrm{i}}{t-t_{*}},
$$


(up to pairwise changes of signs). Similarly, $x$ and $y$ behave like $\epsilon\left(t-t_{*}\right)^{-2}$ and $\epsilon^{2}\left(t-t_{*}\right)^{-3}$, respectively. This behavior is valid for $\epsilon \ll\left|t-t_{*}\right| \ll 1$ and should match the inner solution. This suggests the inner scaling

$$
u=\epsilon^{-1} U(\tau), v=\epsilon^{-1} V(\tau), w=\epsilon^{-1} W(\tau), x=\epsilon^{-1} X(s, \tau) \text { and } y=\epsilon^{-1} Y(s, \tau),
$$

where $\tau$ is a rescaled time defined by

$$
t=t_{*}+\epsilon \tau \text {. }
$$

Introducing this into (2.6)-(2.10) leads to essentially identical equations with lowercase variables replaced by their uppercase counterparts, time derivatives replaced by $\tau$-derivatives, and, crucially, $\epsilon$ set to 1 . This is best solved using the Fourier transform of $X$ and $Y$ or, more conveniently, a small modification thereof. Noting the form (4.3) of $\hat{f}(k)$, we define

$$
\bar{X}=k^{-1} \mathrm{e}^{k^{2} / 2} \hat{X} \text { and } \bar{Y}=k^{-1} \mathrm{e}^{k^{2} / 2} \hat{Y},
$$

where, as before, the hat denotes the Fourier transform in $s$. This leads to the system

$$
\begin{aligned}
U^{\prime} & =-V W-\mathrm{i} a V \int k^{2} \mathrm{e}^{-k^{2}} \bar{Y}(k) \mathrm{d} k, \\
V^{\prime} & =W U+\mathrm{i} a U \int k^{2} \mathrm{e}^{-k^{2}} \bar{Y}(k) \mathrm{d} k, \\
W^{\prime} & =-U V \\
\bar{X}^{\prime} & =-\bar{Y}, \\
\bar{Y}^{\prime} & =\Omega^{2} \bar{X}+\frac{\mathrm{i} a}{2 \pi} U V,
\end{aligned}
$$

where $^{\prime}=\mathrm{d} / \mathrm{d} \tau$ and $\Omega$ is the scaled frequency defined in (4.4). The large- $\tau$ behavior of the solutions, needed for matching, can be obtained by expanding in inverse powers of $\tau$. Specifically, we let

$$
\begin{gathered}
U=\mathrm{i} \sum_{n=1}^{\infty} \frac{U_{n}}{\tau^{2 n-1}}, V=\sum_{n=1}^{\infty} \frac{V_{n}}{\tau^{2 n-1}}, W=\mathrm{i} \sum_{n=1}^{\infty} \frac{W_{n}}{\tau^{2 n-1}}, \\
\bar{X}=\sum_{n=1}^{\infty} \frac{\bar{X}_{n}(k)}{\tau^{2 n}} \text { and } \bar{Y}=\sum_{n=1}^{\infty} \frac{\bar{Y}_{n}(k)}{\tau^{2 n+1}},
\end{gathered}
$$

and obtain a set of recurrence relations for the (real) coefficients $U_{n}, V_{n}$, etc. With the initial conditions

$$
U_{1}=-1, V_{1}=-1, W_{1}=1, \bar{X}_{1}=\frac{a}{2 \pi \Omega^{2}} \text { and } \bar{Y}_{1}=2 \bar{X}_{1},
$$

found by matching with the leading-order outer solution, these recurrence relations can be solved numerically by discretizing the $k$-dependence of $\bar{X}_{n}$ and $\bar{Y}_{n}$ in some way. The integrals in (B.4)-(B.5) can be computed efficiently using Gauss-Hermite quadrature, so we take the discrete values $k_{j}, j=0,1,2, \cdots, M$ of the wavenumber to be the non-negative zeroes of a Hermite polynomial of sufficiently high order $2 M+1$. We thus obtain $2 M+5$ coupled, nonlinear recurrence relations for the coefficients $U_{n}$, $V_{n}, W_{n}, \bar{X}_{n}\left(k_{j}\right)$ and $\bar{Y}_{n}\left(k_{j}\right)$ which we solve numerically. 


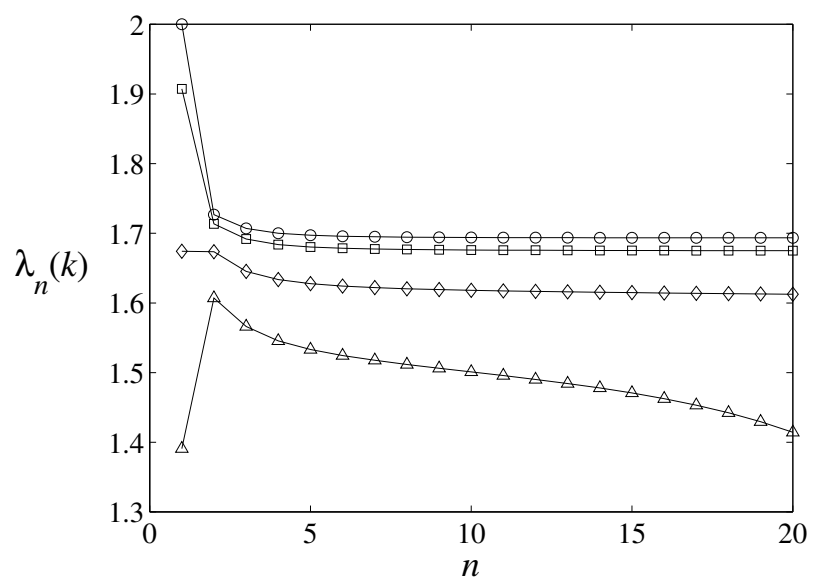

FIG. B.1. Coefficients $\lambda_{n}(k)$ defined in (B.10) as a function of iteration number $n$, for $a=2$ and $b=1$. Four values of $k$ are shown, corresponding to the first four zeroes of the Hermite polynomial $H_{101}$, namely $k_{0}=0(\mathrm{O}), k_{1}=0.22(\square), k_{2}=0.44(\diamond)$ and $k_{3}=0.66(\triangle)$. For $j=0,1,2$, the $\lambda_{n}\left(k_{j}\right)$ converge to finite values $\lambda\left(k_{j}\right)$ as $n \rightarrow \infty$, but $\lambda\left(k_{3}\right)$ diverges (as do $\lambda_{n}\left(k_{j}\right)$ for $j \geq 3$ ).

What matters for the estimation of the Stokes multiplier $C(k)$ is the behavior of these coefficients for $n \gg 1$. We can concentrate on $\bar{X}_{n}$. By analogy with the treatment of the LK model [34], we expect the linear terms in the recurrence to dominate for $n \gg 1$, leading to the asymptotics

$$
\bar{X}_{n} \sim \frac{(-1)^{n+1}(2 n-1) ! \lambda(k)}{2 \pi \Omega^{2 n}},
$$

for some function $\lambda(k)$ which depends on the early-term behaviour and needs to be determined numerically. (The factor $2 \pi$ in the denominator is introduced for convenience.) The numerical determination of $\lambda(k)$ is achieved by computing

$$
\lambda_{n}(k)=\frac{(-1)^{n+1} 2 \pi \Omega^{2 n}}{(2 n-1) !} \bar{X}_{n}
$$

for $n$ large enough. Our computations indicates that the convergence $\lambda_{n}(k) \rightarrow \lambda(k)$ only occurs for $k$ small enough; in other words, the asymptotic behavior (B.9) holds true only for small $k$. This is illustrated by Figure B.1, obtained for $a=2, b=1$ and $M=50$. The divergence for $k$ of order unity occurs because for such $k$, the recurrence relations are not dominated by their linear parts for $n \gg 1$ as is assumed to obtain (B.9). This divergence is inconsequential, however: as shown below, the asymptotics of $x_{\mathrm{w}}$ for $\epsilon \rightarrow 0$ is controlled by the small- $k$ behavior of $\lambda(k)$, indeed by $\lambda(0)$.

Assuming this, we rely on (B.9) and use a Borel-summation technique $[16,17]$ to relate the behavior of $\bar{X}(k, \tau)$ for $\operatorname{Re} \tau<0$ and $\operatorname{Re} \tau>0$. We write (formally)

$$
\bar{X}=\int_{0}^{\infty} \frac{\mathrm{e}^{-s}}{s} B(s / \tau) \mathrm{d} s, \text { where } B(\cdot)=\sum_{n=1}^{\infty} \frac{\bar{X}_{n}}{(2 n-1) !}(\cdot)^{2 n}
$$

and note that the late behavior (B.9) is associated with the existence of a pole of 
$B(s / \tau)$, in the neighborhood of which

$$
B(s / \tau) \sim \frac{\lambda(k) s^{2}}{2 \pi\left(s^{2}+\Omega^{2} \tau^{2}\right)} .
$$

This pole yields an oscillatory (or wave) contribution to (B.11) for $\operatorname{Re} \tau>0$, i.e. after the Stokes line is crossed. Evaluating this leads to the wave part of $\bar{X}$ in the form

$$
\bar{X}_{\mathrm{w}}=-\frac{\mathrm{i} \lambda(k)}{2} \mathrm{e}^{-\mathrm{i} \Omega \tau}=-\frac{\mathrm{i} \lambda(k)}{2} \mathrm{e}^{-\mathrm{i} \omega\left(t-t_{*}\right)},
$$

with $\omega>0$. Taking into account the contribution of the complex-conjugate pole $\bar{t}_{*}$, and matching with (B.3) gives the wave amplitude (4.8).

Multiplying (4.8) by $\exp (\mathrm{i} k s)$ and integrating over $k$ then gives an estimate for $x_{\mathrm{w}}$. The smallness of $\epsilon$ can be used to approximate the resulting integral using the steepest-descent method. The saddle is located at $k=0$, indicating that wavenumbers $k=O\left(\epsilon^{1 / 2}\right)$ dominate the integral. This suggests the natural rescaling of the spatial variable as $S=\epsilon^{1 / 2} s$. Assuming this to be $O(1)$, we obtain (4.9). Note that an estimate valid uniformly in $s$ could be obtained by including the term iks in the saddle-point calculation.

\section{REFERENCES}

[1] M. J. Ablowitz And A. S. Fokas, Complex variables: introduction and applications, Cambridge University Press, 1997.

[2] M. Abramowitz and I. A. Stegun, Handbook of mathematical functions, Dover, 1965.

[3] M. V. BerRY, Uniform asymptotic smoothing of Stokes's discontinuities, Proc. R. Soc. Lond. A, 422 (1989), pp. 7-21.

[4] L. Blanchet, Gravitational radiation from post-Newtonian sources and inspiralling compact binaries, Living Rev. Relativity, 5 (2002), p. $3 . \quad$ online article, http://www.livingreviews.org/lrr-2002-3.

[5] O. BoKHOvE, Balanced models in geophysical fluid dynamics: Hamiltonian formulation and formal stability, in Large-scale atmosphere-ocean dynamics, I. Roulstone and J. Norbury, eds., vol. II: Geometric methods and models, Cambridge University Press, 2002, pp. 1-63.

[6] O. Bokhove And T. G. Shepherd, On Hamiltonian balanced dynamics and the slowest invariant manifold, J. Atmos. Sci., 53 (1996), pp. 276-297.

[7] T. J. BRIDges AND S. ReICH, Multi-symplectic integrators: numerical schemes for hamiltonian pdes that conserve symplecticity, Phys. Lett. A, 284 (2001), pp. 183-193.

[8] R. CAmassa, On the geometry of an atmospheric slow manifold, Physica D, 84 (1995), pp. 357397.

[9] R. Daley, Atmospheric data analysis, Cambridge University Press, 1991.

[10] A. Einstein, Über Gravitationswellen, Sitzunberg. K. Preuss. Akad. Wiss., (1918).

[11] R. FORD, Gravity wave radiation from vortex trains in rotating shallow water, J. Fluid Mech., 281 (1994), pp. 81-118.

[12] R. Ford, M. E. McIntyre, And W. A. Norton, Balance and the slow quasi-manifold: some explicit results, J. Atmos. Sci., 57 (2000), pp. 1236-1254.

[13] — Reply to comments by S. Saujani and T. G. Shepherd on "Balance and the slow quasimanifold: some explicit results", J. Atmos. Sci., 59 (2002), pp. 2878-2882.

[14] V. Gelfreich and L. Lerman, Almost invariant elliptic manifold in a singularly perturbed Hamiltonian system, Nonlinearity, 15 (2002), pp. 447-457.

[15] T. Hagstrom, Radiation boundary conditions for the numerical simulation of waves, Acta Num., 8 (1999), pp. 47-106.

[16] V. HAKIM, Computation of transcendental effects in growth problems: linear solvability conditions and nonlinear methods - the example of the geometric model, in Asymptotics beyond all orders, H. Segur, S. Tanveer, and H. Levine, eds., Plenum press, 1991, pp. 15-28.

[17] _ - Asymptotic techniques in nonlinear problems: some illustrative examples, in Hydrodynamics and nonlinear instabilities, C. Godrèche and P. Manneville, eds., Cambridge University Press, 1998, ch. 3, pp. 295-386. 
[18] L. D. Landau and E. M. Lifschitz, The classical theory of fields, Pergamon, 4th ed., 1975.

[19] - Fluid Mechanics, Pergamon, 2nd ed., 1987.

[20] M. J. Lighthill, On sound generated aerodynamically, I. General theory, Proc. R. Soc. Lond. A, 211 (1952), pp. 564-587.

[21] E. N. Lorenz, On the existence of a slow manifold, J. Atmos. Sci., 43 (1986), pp. 1547-1557.

[22] E. N. Lorenz and V. Krishnamurthy, On the nonexistence of a slow manifold, J. Atmos. Sci., 44 (1987), pp. 2940-2950.

[23] P. LYNCH, The swinging spring: a simple model for atmospheric balance, in Large-scale atmosphere-ocean dynamics, I. Roulstone and J. Norbury, eds., vol. II: Geometric methods and models, Cambridge University Press, 2002, pp. 64-108.

[24] R. S. MacKay, Slow manifolds, in Energy Localisation and Transfer, T. Dauxois, A. LitvakHinenzon, R. S. MacKay, and A. Spanoudaki, eds., World Sci., 2004, pp. 149-192.

[25] M. E. MCIntyre and I. Roulstone, Are there higher-accuracy analogues of semi-geostrophic theory?, in Large-scale atmosphere-ocean dynamics, I. Roulstone and J. Norbury, eds., vol. II: Geometric methods and models, Cambridge University Press, 2002, pp. 301-364.

[26] J. C. McWilliams, J. M. Molemaker, and I. Yavneh, From stirring to mixing of momentum: Cascades from balanced flows to dissipation in the oceanic interior., in Aha Huliko'a Proceedings 2001, U. of Hawaii, 2001, pp. 59-66.

[27] E. I. Ólafsdóttir, A. B. O. Dahlhuis, and J. Vanneste, Multiple Stokes multipliers in a inhomogeneous differential equation with a small parameter, Proc. R. Soc. Lond. A, 461 (2005), pp. 2243-2256.

[28] R. B. Paris And A. D. Wood, Stokes phenomenon demystified, Bull. Inst. Math. Appl., 31 (1995), pp. 21-28.

[29] G. M. Reznik, V. Zeitlin, And M. B. Jelloul, Nonlinear theory of geostrophic adjustment. Part 1. Rotating shallow-water model, J. Fluid Mech., 445 (2001), pp. 93-120.

[30] C. W. Rowley and T. Colonius, Discretely non-reflecting boundary conditions for linear hyperbolic systems, J. Comput. Phys., 157 (2000), pp. 500-538.

[31] S. Saujani And T. G. Shepherd, Comments on "Balance and the slow quasi-manifold: some explicit results", J. Atmos. Sci., 59 (2002), pp. 2874-2877.

[32] H. Segur, S. Tanveer, and H. Levine, eds., Asymptotics beyond all orders, New York, 1991, Plenum Press.

[33] N. G. van Kampen, Elimination of fast variables, Phys. Rep., 124 (1985), pp. 69-160.

[34] J. VANNESTE, Inertia-gravity-wave generation by balanced motion: revisiting the LorenzKrishnamurthy model, J. Atmos. Sci., 61 (2004), pp. 224-234.

[35] J. VANNESTE AND I. YAVNeH, Exponentially small inertia-gravity waves and the breakdown of quasi-geostrophic balance, J. Atmos. Sci., 61 (2004), pp. 211-223.

[36] T. Warn, O. Bokhove, T. G. Shepherd, and G. K. Vallis, Rossby number expansions, slaving principles, and balance dynamics, Quart. J. R. Met. Soc., 121 (1995), pp. 723-739.

[37] G. B. Whiтнам, Linear and nonlinear waves, Wiley, 1974.

[38] D. Wirosoetisno, Exponentially accurate balance dynamics, Adv. Diff. Eq., 9 (2004), pp. 177196.

[39] D. Wirosoetisno And T. G. Shepherd, Averaging, slaving and balance dynamics in a simple atmospheric model, Physica D, 141 (2000), pp. 37-53. 